Research Article

\title{
Flow Structures of the Air-Water Layer in the Free Surface Region of High-Speed Open Channel Flows
}

\author{
Feng Jiang, Weilin Xu, Jun Deng, and Wangru Wei $i$ \\ State Key Laboratory of Hydraulics and Mountain River Engineering, Sichuan University, Chengdu 610065, China \\ Correspondence should be addressed to Wangru Wei; weiwangru@scu.edu.cn
}

Received 31 May 2020; Revised 22 September 2020; Accepted 21 October 2020; Published 3 November 2020

Academic Editor: George S. Dulikravich

Copyright (C) 2020 Feng Jiang et al. This is an open access article distributed under the Creative Commons Attribution License, which permits unrestricted use, distribution, and reproduction in any medium, provided the original work is properly cited.

\begin{abstract}
In hydraulic engineering, intense free surface breakups have been observed to develop in high-speed open channel flows, resulting in a mixed air-water layer near the free surface that grows with the development of self-aeration. This region is characterized by a substantial number of droplets coexisting with an induced air layer above. Little information about this droplet layer is currently available and no practicable approach has been proposed for predicting the parameters of the induced air layer based on the related flow structures in the droplet layer. In this research, laboratory experiments were accordingly conducted to observe the detailed droplet layer development in terms of layer thickness, droplet size, and frequency distributions under comparative flow conditions. Based on the simplified droplet layer roughness determined using the experimentally measured mean droplet size, the classical power-law of boundary layer theory was applied to provide an analytical solution for the air velocity profile inside the air layer. The relationship of air layer growth to droplet layer thickness, which is a key factor when determining the air velocity distribution, was also established, and the analytical results were proven to be in reasonable agreement with air velocity profiles presented in the literature. By determining the relationship between droplet layer properties and air velocity profiles, the study establishes a basis for the improved modeling of high-speed open channel flows.
\end{abstract}

\section{Introduction}

Large spillways and discharge tunnels are commonly set in high dams and other hydraulic engineering projects. As the water flow velocity increases above a critical condition in such structures, an air-water mixture can be observed to develop downstream in the free surface region. Unlike for a single phase flow, two-phase structures of high-speed airwater flows in open channels broadly consist of two features: air bubbles and water droplets. The different effects of these features are of utmost importance for hydraulic designs considering flow depth, cavitation damage, energy dissipation, and gas-phase transfer. However, it is difficult to unify the free surface air entrainment with the water droplet ejection mechanisms to predict the development of the airwater mixture. This results in a lack of understanding of the stratified flow characteristics in the free surface region.

For the nonaeration region in hydraulic facilities, a clear air-water interface forms the boundary of the stratified flow, and different scales and features of geometric waves are generated for nonaerated flow conditions [1,2]. Detailed particle image velocimetry measurements and statistical theory have implied considerable fluctuation of surface waves above the bulk flow as the air-water boundary is fully developed [3]. As turbulence is sufficiently strong to overcome gravity and surface tension effects, the intact free surface is then broken up and local water bodies are ejected from it $[4,5]$, leading to a "droplet layer" in self-aerated flows [6]. Wilhelms and Gulliver [7] pointed out two air-water structures, implying the coexistence of individual particles and large-scale wave roughness in the free surface region. Because significant air motion results from the turbulence of water-dominated structures, the droplets and water waves induce an air flow above the bulk flow. It is evident that this two-phase layer constitutes a connected surface transition from the water region to the air region. Basic theoretical analyses have determined the turbulence condition for free surface spraying and discontinuity properties, and the airwater surface layer has been identified as an essential factor when determining an appropriate boundary condition for 
open channel flow [8-10]. However, the flow structures in the air-water boundary layer, where both air and water interact and interpenetrate, are less well known than the classical wall boundary structures.

As air-water flows are generated by open channel flows, the characterized depth $y_{90}$, at which the local air concentration $C$ is 0.90 , is considered to be the flow depth. The region above $y_{90}$ is the air-water layer, primarily characterized by a droplet layer and surface wave fluctuations, as shown in Figure 1. Analogous to the development of the turbulent boundary layer in chute flows [11], the droplet layer coexists with an interaction region of complex flow structures above the free surface at $y_{90}$. This mixed air and water boundary layer is generally consistent with traditional boundary layer theory $[12,13]$. The air flow generated above an aerated flow is mainly driven by the upper mixture boundary layer, but the prediction of droplet layer behavior is necessary to determine the boundary shear effect. Valero and Bung [14] provided a systemic theoretical analysis of the air flow development over a free surface, suggesting the effect of detailed wave amplitudes on the prediction of air velocity and air boundary layer thickness. Previous experimental studies using two-phase flow measurements have also indicated a series of air-water structures in the mainly aerated region below $y_{90}$ [15-17]. These detailed flow structures are complex and exist in different aeration levels, including a wide range of individual droplets and bubbles, air-water projections, and large-scale waves. It has accordingly been theorized that an analysis of air flow layer development should take the noncontinuous features of the droplet layer into consideration [18]. However, there remains a lack of information describing the flow structures of the air-water layer in the free surface region. Thus, comprehension of droplet layer boundary conditions in the free surface region is of paramount importance for understanding air flow phenomena under these conditions.

To the authors' knowledge, the effects of stratified flow structures and the air-water interface drag effect on open channel flows and the effect of droplet layer development on the air flow over open channel flows remain undetermined. The objective of the present study is therefore to investigate and present the detailed features of droplet layer development. An improved approach for the air velocity distribution dragged by the droplet layer is proposed based on traditional analytical solutions. The developed model is demonstrated to apply to the description of the air flow phenomenon above the highly aerated and rough free surface region of open channel flows.

\section{Methods and Boundary Considerations}

2.1. Hydraulic Model and Experimental Methods. All experiments were conducted using a smooth chute with a constant slope $\alpha$ of $28^{\circ}$. The chute channel was $0.30 \mathrm{~m}$ wide and $18 \mathrm{~m}$ long. The bottom floor of the channel was made of steel plates, and the side-walls were made of glass. A jet box was set at the origin to release water into the smooth channel, enabling control of the approach flow condition. The nozzle height was adjustable to control the initial flow depth $h_{0}$. The water flow discharge was supplied by a water circuit system and controlled by a thin-plate weir downstream. The water flow discharge per unit width $q_{\mathrm{w}}$ was $0.31-0.84 \mathrm{~m}^{2} / \mathrm{s}$, resulting in air-water flow velocities around $6.0-12.0 \mathrm{~m} / \mathrm{s}$ in all tested regions. The approaching flow Reynolds number $\operatorname{Re}=\left(V_{0} / h_{0}\right) / U$ was approximately $3.1 \times 10^{5}-8.4 \times 10^{5}$, where $V_{0}$ is the averaged flow velocity at the jet box deduced from $V_{0}=q_{w} / h_{0}$. Eight cross-sections along the chute were plotted to capture the development process of the air-water mixture in the free surface region of the high-speed open channel flow. The coordinate $x$ is defined as the streamwise direction along the channel bottom, where $x=0$ is the initial cross-section at the self-aeration inception point. The coordinate perpendicular to $x$ is $y$, and the channel bottom slope $\alpha$ is constant with a constant channel width along $x$ direction. In the present experimental condition, as the flow velocity at the initial cross-section connected to the jet box is higher than $6 \mathrm{~m} / \mathrm{s}$, the free surface air entrainment is obvious and it is considered as the selfaeration inception point. The dimensionless parameter $x / h_{0}$ is used to describe the various cross-sections along the development process.

Air-water layer velocities and interface transfer were recorded using a double-tip conductivity probe (CQY-Z8a instrument), as shown in Figure 2. Each probe consisted of two identical concentric tips with a $0.04 \mathrm{~mm}$ diameter internal platinum electrode and a $0.7 \mathrm{~mm}$ diameter external stainless steel electrode. The probe tips were aligned in the flow direction, and the two tips were placed $\Delta l=9.5 \mathrm{~mm}$ apart. Previous model experiments and prototype measurements, and the phase-detection probes were confirmed as the effective measurement method in air-water mixture flows $[19,20]$. The air-water interface signal quality was determined by the needle diameter size, scan rate, and period. Firstly, the smallest particle (water droplets and air bubbles) size resolution of this measurement was closely linked to the needle sensor diameter size, which needed to be smaller than the size resolution. As the internal platinum diameter was $0.04 \mathrm{~mm}$, the smallest particle size resolution was determined as $0.05 \mathrm{~mm}$. With a maximum air-water flow velocity $9 \mathrm{~m} / \mathrm{s}$ and a resolution size $0.05 \mathrm{~mm}$, the scan rate should be greater than $9(\mathrm{~m} / \mathrm{s}) / 0.05(\mathrm{~mm})=180 \mathrm{kHz}$. Moreover, the air concentration (in the high air concentration area), water droplet frequency $F$, and number mean droplet size $d_{w m}$ were almost stable at a certain measurement point at a series of scan period ( $T \geq 5 \mathrm{~s})$, as shown in Figure 3. Thus, the signals from the conductivity probe were recorded at a scan rate of $F_{\text {sample }}=200 \mathrm{kHz}$ per channel for a scan period of $T=5 \mathrm{~s}$. The results reported in this study were computed in terms of the probability of encountering the air-water interface at the leading tip of the probe. Velocity measurements were then based on two successive detections of the air-water interface by the two tips.

2.2. Air-Water Interface Layer Considerations. The typical air concentration distribution in a self-aerated open channel flow is hypothesized to present a continuous profile increasing from a certain value at the chute bottom 


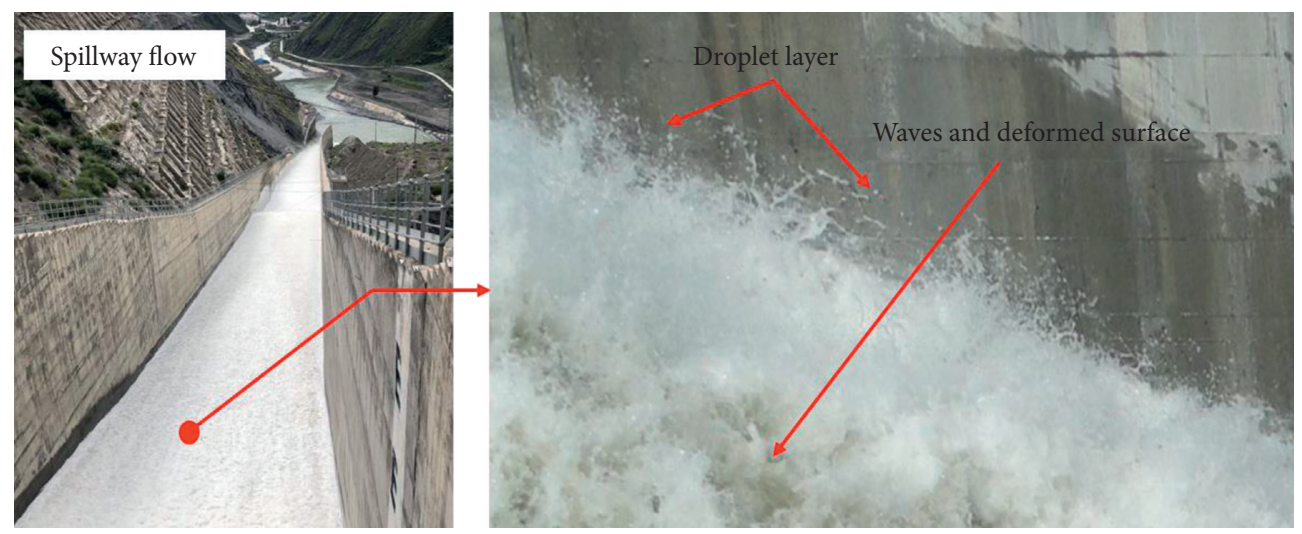

FIGURE 1: Air-water mixture of free surface region in self-aerated open channel flows.
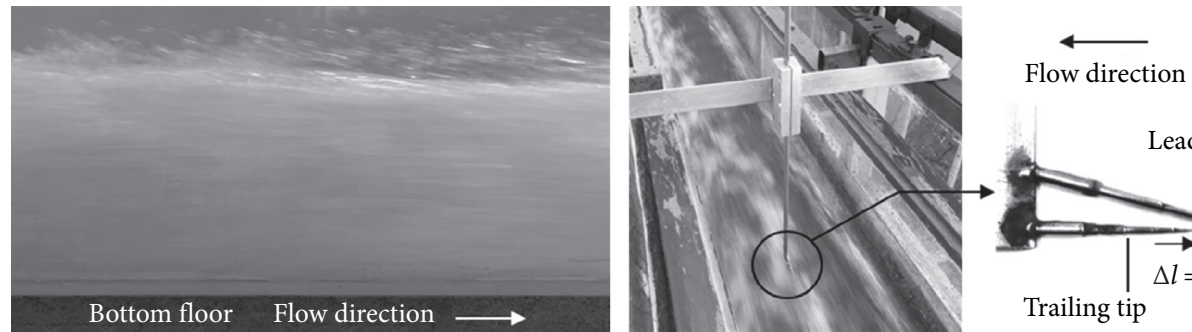

Figure 2: Air-water mixture of free surface region in self-aerated open channel flows.

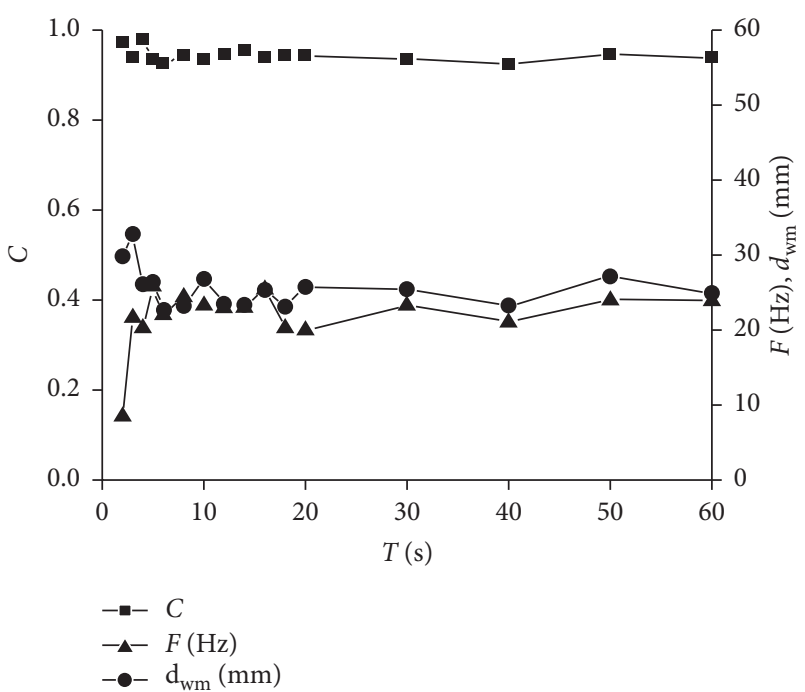

FIGURE 3: Effects of scan rate and period on the measurement $\left(V_{0}=9.0 \mathrm{~m} / \mathrm{s} ; d_{0}=0.05 \mathrm{~m}\right)$.

to clear air with a $C$ of 1.0. In this study, the characterized depth $y_{90}$ is considered to be the free surface location in the air-water bulk flow with $y$ being the coordinate perpendicular to the chute bottom (Figure 4). Given that there is no velocity slip between air and water at $y_{90}$, the average free surface velocity is $u_{\mathrm{fs}}$. The droplet layer is above $y_{90}$, and its thickness $\delta_{\mathrm{w}}$ is obtained by $=y_{99}-y_{90}$, where $y_{99}$ is the characterized flow depth with $C=0.99$. The average airwater velocity in the droplet layer thickness is $u_{\mathrm{w}}$, representing the drag source. Due to the shear compatibility of the air and water phases in the free surface region, air velocity $\left(u_{\mathrm{a}}\right)$ gradients develop in the vertical direction. This results in an air flow layer with a thickness of $\delta_{\mathrm{a}}$ above the droplet layer. The vertical span $y_{\mathrm{a}}$ of this air flow layer thus ranges from the upper boundary of the droplet layer at $y_{99}$ to $y_{99}+\delta_{\mathrm{a}}$. As the initial flow velocity in all tests exceeded $6 \mathrm{~m} / \mathrm{s}$, the dimensionless parameter $x / h_{0}$ is used to describe the various cross-sections along the development process, where $x$ is the coordinate along the direction of flow at the chute bottom.

The following definitions are used for droplet description in the present analysis. An individual droplet is considered an intact volume of water entity and is detected by the leading tip of the probe as two consecutive signals of airwater interface transfer events. The size of an individual droplet $d_{\mathrm{w}}$ is defined as the droplet chord size, which is the length of the straight distance between two consecutive airwater interface intersections. Thus, the droplet is not the form of droplets surrounded by air. For a total water droplet sample number $N$ in the scan period, the droplet frequency $F$ is the sample number of droplets per unit time, and the number mean droplet size $d_{\mathrm{wm}}$ is defined as

$$
d_{\mathrm{wm}}=\frac{\sum_{i=1}^{N} n_{i}\left(d_{\mathrm{w}}\right)_{i}}{\sum_{i=1}^{N} n_{i}},
$$

where $n_{i}$ is the number of droplets of a certain size $\left(d_{\mathrm{w}}\right)_{i}$. The mean droplet size across the entire droplet layer is defined as

$$
D_{\mathrm{wm}}=\frac{1}{\delta_{\mathrm{w}}} \int_{y_{90}}^{y_{99}} d_{\mathrm{wm}} \mathrm{d} y .
$$



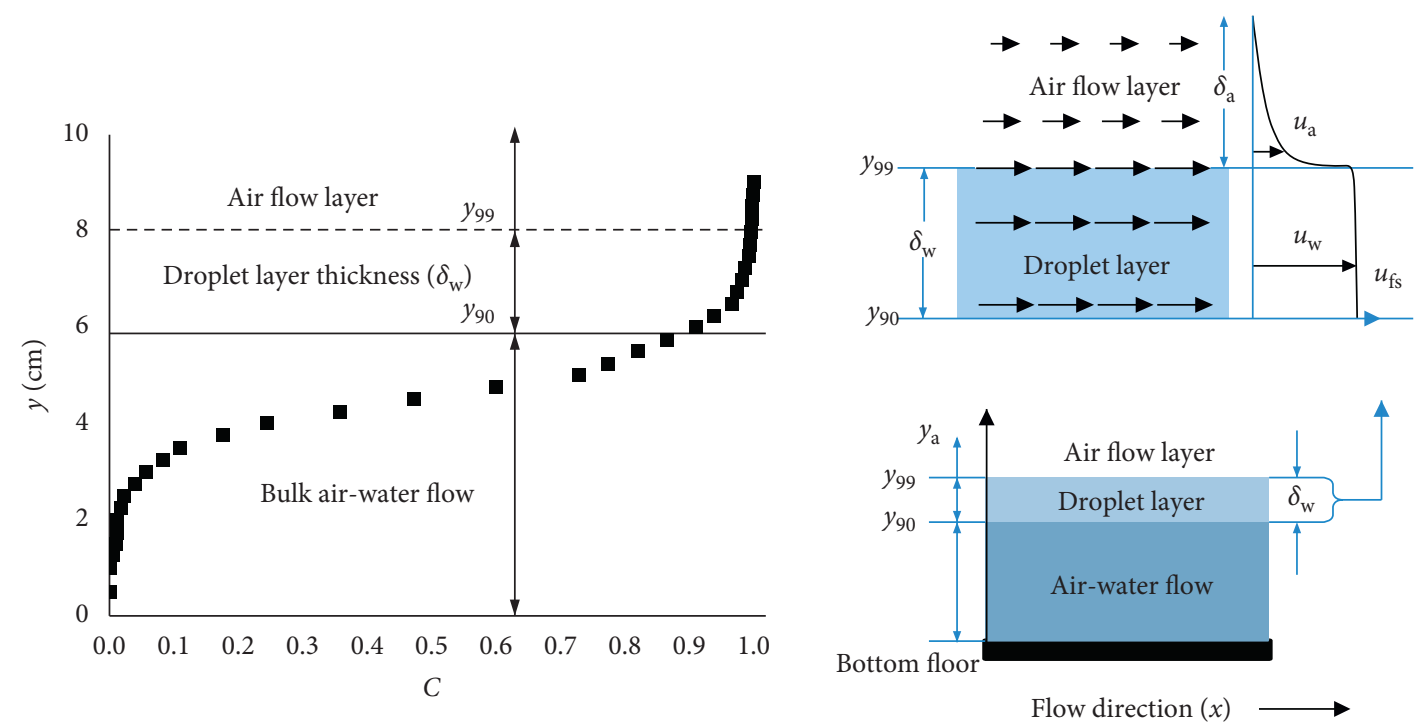

Air concentration

FIGURE 4: Sketch of air-water layer development near the free surface region.

It should be noted that as the probe represents an intrusive measurement, its tips measure the water droplet chord length, not the exact droplet diameter. Water droplets are not uniformly sized as spherical particles, but at a given location in the air-water mixture layer, the droplet chord length is considered in this study to be proportional to the droplet diameter to provide a description of water droplet size scale.

According to the classical model of a turbulent boundary layer in chute flow, such as the wall-wake model [12], the equivalent roughness height in the interface region (e.g., wall roughness height) is a critical component in the descriptions of the boundary layer growth and relative velocity profile. Moreover, the thickness of the droplet layer is a key factor determining the strength of the drag effect. As the droplet layer is generated by an increase in bulk flow development, a thicker droplet layer causes a stronger drag effect on the air flow layer above. In Section 3, data describing the characteristics of droplets in the droplet layer are first analyzed to illustrate the droplet layer variation with the development of self-aeration and to generalize droplet roughness in the air-water interface layer. On the basis of this simplified description of droplet layer thickness and roughness, an improved analysis model for air velocity distribution prediction is then proposed. Finally, the experimental data from previous investigations are used to validate the improved model.

\section{Results and Discussion}

3.1. Droplet Layer Development. Figure 5 shows the development of water droplet layer thickness in a self-aerated open channel flow. To derive $\delta_{\mathrm{w}} / y_{90}$ as a function of $x / h_{0}$, the experimental results and prototype data from different literature $[6,21,22]$ are considered. Detailed hydraulic conditions for theses air-water mixture measurement are shown in Table 1 . The value of $\delta_{\mathrm{w}} / y_{90}$ is 0 at the inception point

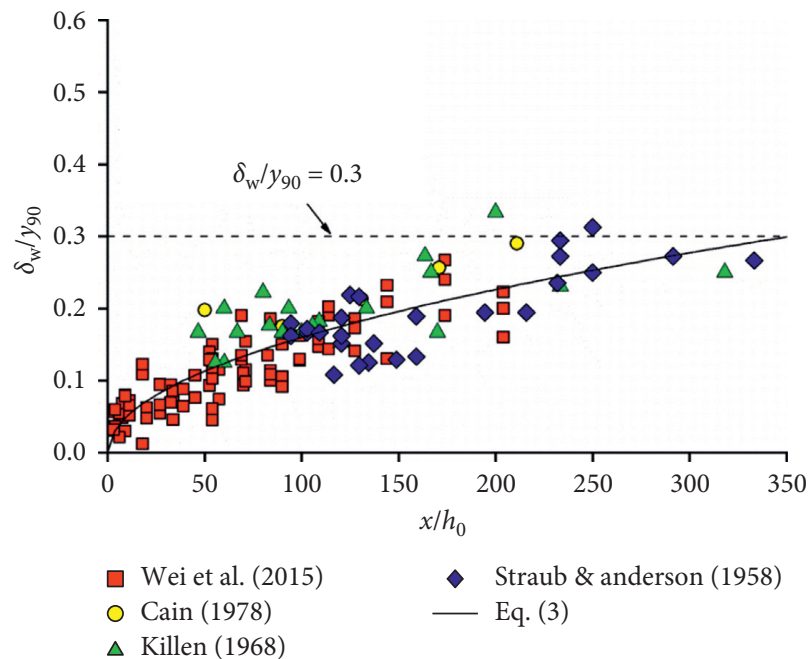

Figure 5: Development of water droplet layer thickness.

where self-aeration occurs. The data analysis indicates that the relationship between $\delta_{\mathrm{w}} / y_{90}$ and $x / h_{0}$ satisfies selfsimilarity and that the increase in $\delta_{\mathrm{w}} / y_{90}$ is independent of other flow conditions. The power-function approximation is thus applied as follows:

$$
\frac{\delta_{\mathrm{w}}}{y_{90}}=\eta \cdot\left(\frac{x}{h_{0}}\right)^{0.5},
$$

where the relevant parameter $\eta$ from the data analysis is suggested as $\eta=0.016$, resulting in a coefficient of determination $R^{2}=0.8615$ for this approximation. As self-aeration develops downstream around $x / h_{0}=200-300$, the thickness of the droplet layer can reach about $30 \%$ of the bulk flow depth. Note that equation (3) can be applied for $0 \leq\left(x / h_{0}\right) \leq 350,0.1 \leq q_{\mathrm{w}} \leq 3.2 \mathrm{~m}^{2} / \mathrm{s}$, and $7.5^{\circ} \leq \alpha \leq 75^{\circ}$. As it is similar to the uniform trend of wall boundary layer growth 
TABLE 1: Hydraulic conditions for air-water mixture measurement in self-aerated flows.

\begin{tabular}{lcccc}
\hline References & $q_{\mathrm{w}}\left(\mathrm{m}^{2} / \mathrm{s}\right)$ & $\alpha$ & Aeration state & Comments \\
\hline Straub \& Anderson [6] & $0.1-0.9$ & $7.5^{\circ}-75^{\circ}$ & Developing \& equilibrium & Models, electrical conductivity probe. \\
Killen [21] & $0.4-0.9$ & $30^{\circ}-52.5^{\circ}$ & Developing & Prototype, phase-detection probe. \\
Cain and Wood [22] & $2.2-3.2$ & $45^{\circ}$ & Developing & Models, phase-detection probe. \\
Present study & $0.3-0.8$ & $28^{\circ}$ & Developing & \\
\hline
\end{tabular}

inside the flow $[11,23,24]$, the uniform trend of droplet layer growth proves that a universal model can be constructed for air velocity profile generation.

3.2. Droplet Characteristics in Droplet Layer. Figure 6 shows the droplet frequency distribution above the free surface $y_{90}$, where $F_{90}$ is the droplet frequency at the free surface. The droplet frequency gradually decreases with the increase in the distance above the free surface, approximately following a power-law trend. For the initial cross-section at $0 \leq x / h_{0} \leq 10$, the decrease gradient of $F / F_{90}$ with $y / y_{90}$ is considerable due to the weak generation of the air-water mixture at the free surface. As self-aeration develops downstream, this decrease slows, indicating a stronger generation of the air-water mixture. The breakup of large waves into small droplets results in the ejection of more droplets from the bulk air-water flow. The relationship between $y / y_{90}$ and $F / F_{90}$ can therefore be written as

$$
\frac{y}{y_{90}}=\left(\frac{F}{F_{90}}\right)^{-m}
$$

where the data obtained in this study show that the power coefficient $m$ is 0.02 if $0 \leq x / h_{0} \leq 10,0.06$ if $50 \leq x / h_{0} \leq 90$, and 0.12 if $100 \leq x / h_{0} \leq 210$. There is an obvious difference in droplet frequency distribution at certain cross-sections for different $\mathrm{Re}_{0}$ conditions, as shown in Figure $7(\mathrm{a})$, but $\mathrm{Re}_{0}$ appears to have little effect on the droplet size distribution in the droplet layer under these flow conditions. In Figure 7(b), the water-dominant structure size in the near free surface region $\left(y / y_{90}<1.05\right)$ can be observed to be an order of magnitude larger than the droplet layer thickness $\delta_{\mathrm{w}}$. A boundary condition for (4) is $F / F_{90}=1$ for $y / y_{90}=1$. The excessive accumulation variation in the near free surface closed to $y / y_{90}=1$ represents the air-water structures are mainly large scales, such as distorted surfaces and waves. For high self-aeration area $(C>0.9)$, the air-water structures are much complicated and not the form of droplets surrounded by air. In the near free surface region, the air-water structures are large scales, such as distorted surfaces and waves, and the value of $d_{\mathrm{wm}} / \delta_{\mathrm{w}}$ is mainly greater than 1.0. In the upper region $\left(y / y_{90}>1.05\right)$, the droplet size scale decreases significantly, reaching about $0.5 \delta_{\mathrm{w}}$. This indicates that these large structures break up into much smaller scales, such as individual droplets, high sprays, foams, and air-water projections.

The violent turbulence of a convex free surface has been observed to dominate splitting droplet evolution in the airwater interface region [25-27]. Observations of individual droplets ejected by turbulent wall jets have indicated that the terminal droplet size moving above the free surface is about 0.4-0.8 times the jet size [28]. Additionally, the droplet

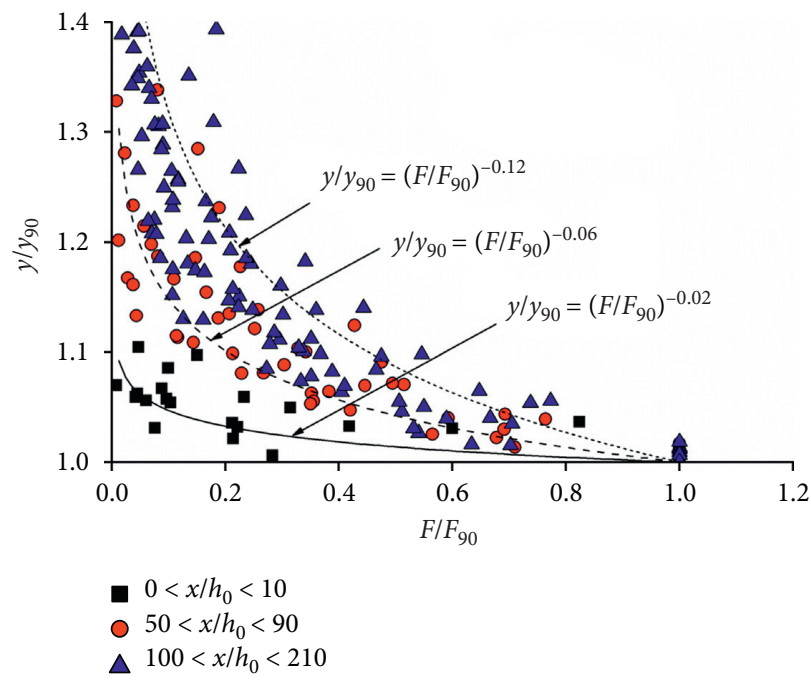

FIGURE 6: Droplet frequency distribution above the free surface $\left(\operatorname{Re}_{0}=4.5 \times 10^{5}\right)$.

characteristics in the spray layer are independent of the Reynolds number, Weber number, and wall roughness conditions. The geometrical similarity of droplets in the droplet layer of an open channel flow indicates a universal mechanism of free surface momentum transfer. The characteristic droplet size can therefore be represented by the droplet layer thickness, compared to wall jets in previous studies. Thus, the traditional boundary layer model is applicable to the generation of the droplet layer and its geometrical development.

In the droplet layer development region of the present study, the average droplet size $D_{\mathrm{wm}}$ increases over the original development distance $(x=2-3 \mathrm{~m})$, reaching around $10-15 \mathrm{~mm}$ as shown in Figure 8. The average droplet size then decreases to around 5-10 $\mathrm{mm}$ with the development of self-aeration downstream. This indicates that although large air-water structures dominate in a specific region close to the free surface, the quantitative domination of small air-water structures leads to a stable droplet size. Indeed, the water droplets evolving from the free surface mass transfer had sizes of 1-10 $\mathrm{mm}$ in the droplet layer of the self-aerated open channel flows evaluated in this study. The results of the present study thus indicate that the extremes of the droplet size scale are independent of the effect of turbulence on the dynamic air-water mixture.

An approximation of the flow velocity gradient in the droplet layer is very important when considering the air flows induced by water droplets. The measurement of airwater flow velocity expresses a slightly decreasing trend in the vertical direction in Figure 9(a). It is therefore assumed 


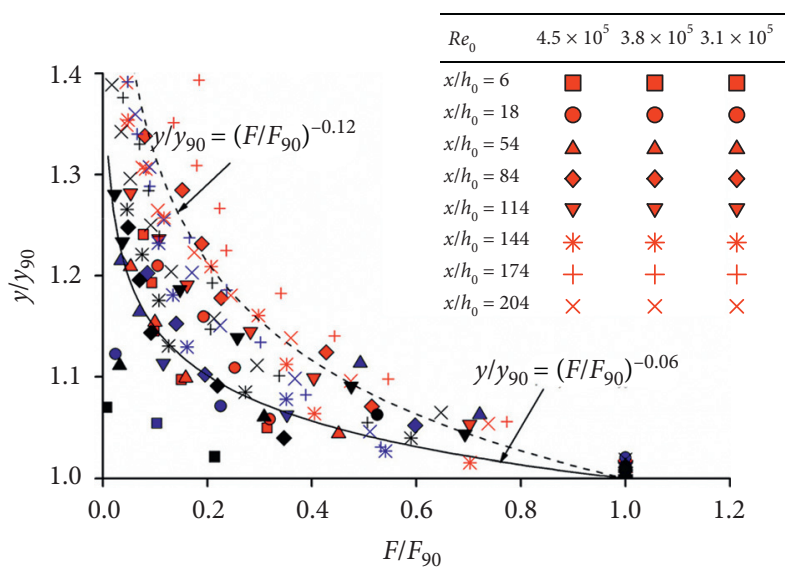

(a)

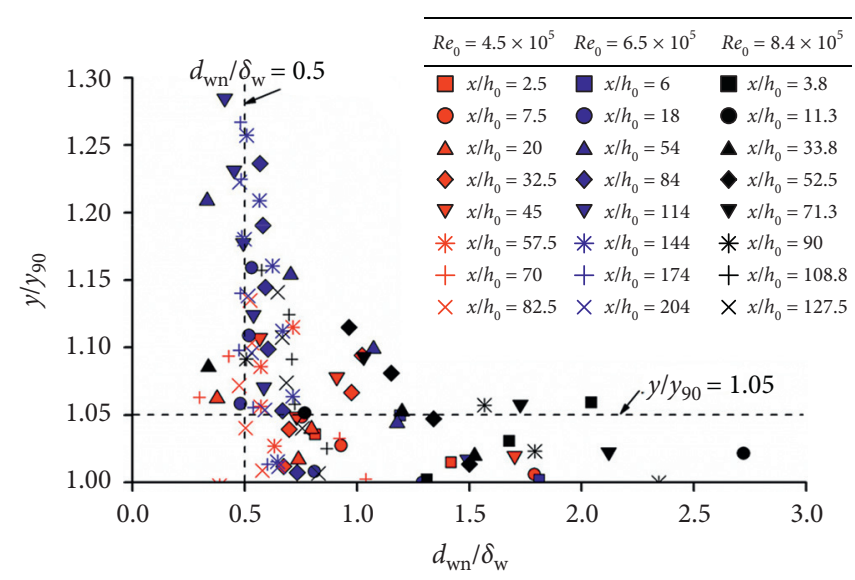

(b)

Figure 7: Effects of flow Reynolds number on (a) droplet frequency and (b) size scale distributions above the free surface.

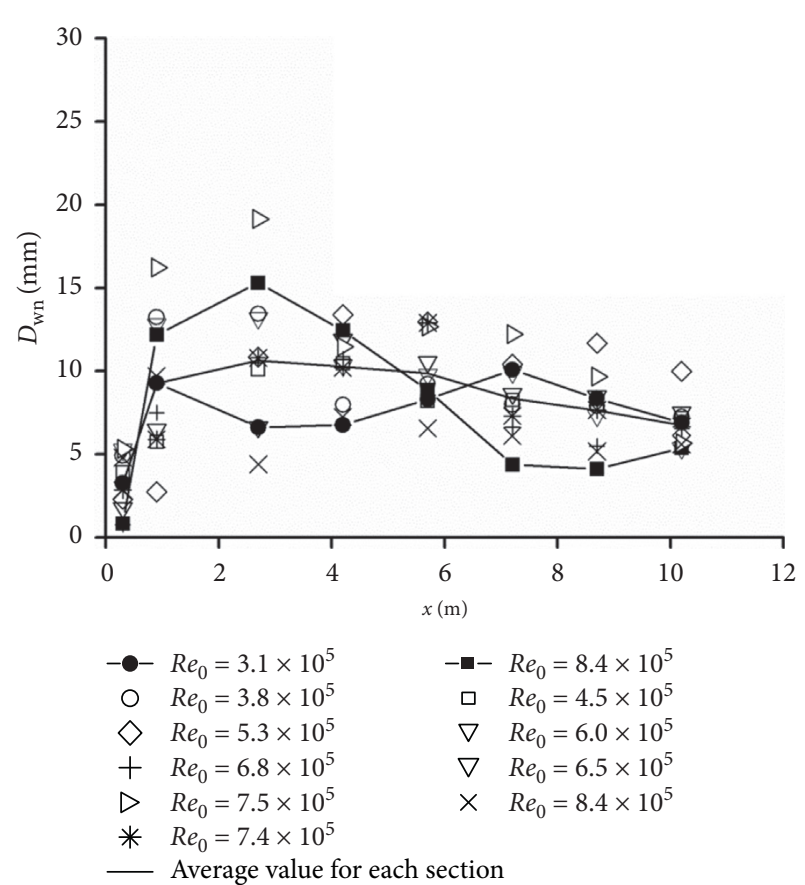

FIGURE 8: Streamwise variation of cross-sectional mean droplet sizes.

that the terminal decrease in the velocity profile occurs in both the air and air-water mixture area. The empirical velocity gradient in the droplet layer is accordingly estimated to be $u_{\mathrm{fs}}-u_{\mathrm{w}} \approx 0.10 u_{\mathrm{fs}}$ for $y=\delta_{\mathrm{w}}$. The formation process of water droplets is affected by the turbulence effect overcoming the surface tension. Using the characteristic dimension $\delta_{\mathrm{w}}$ and free surface velocity $u_{\mathrm{fs}}$, a characteristic Weber number, $\mathrm{We}$, is introduced as $\mathrm{We}=\left(\rho D_{\mathrm{wm}} u_{f s} 2\right) / \sigma$, where $\rho$ is the water density and $\sigma$ is the surface tension $(0.072 \mathrm{~N} / \mathrm{m})$. The correlation of the present measurements $D_{\mathrm{wm}} / \delta_{\mathrm{w}}$ as a function of We are plotted in Figure 9(b). A similar relationship, $D_{\mathrm{wm}} / \delta_{\mathrm{w}}=2.8 \cdot \mathrm{We}^{-(3 / 5)}$, is in agreement with an earlier correlation for round free jets and plane wall jets $[29,30]$. With the development of free surface breakup and air-water mixture downstream, the effect of surface tension gets weak and the aspect ratios of $D_{\mathrm{wm}} / \delta_{\mathrm{w}}$ decreases from the approximately same size scale to an order of magnitude smaller. It is also helpful that the present study extends the phenomena cognition of water droplet breakup and air-water mixture at the free surface of high-speed flows.

3.3. Air Velocity Profile. Under the shear stress continuity with the no velocity slip hypothesis, the air movement induced above the bulk air-water flow is assumed to develop along the entire flow. Indeed, an increase in water flow discharge has been observed to result in higher air flow velocity in discharge tunnels and spillways. For engineering applications, formulations should be developed to determine the air layer thickness and related air velocity distribution. Previous studies have indicated that air layer growth can be appropriately described using boundary layer theory [31-33]. Accordingly, the friction factor for air layer growth is defined as

$$
C_{f}=\frac{\tau_{\mathrm{m}}}{0.5 \rho_{\mathrm{m}} u_{f s}^{2}},
$$

in which the $\tau_{\mathrm{m}}$ is the shear stress in the droplet layer:

$$
\tau_{\mathrm{m}}=\mu_{\mathrm{m}} \frac{\partial u_{\mathrm{m}}}{\partial y},
$$

where $\mu_{\mathrm{m}}$ is the dynamic viscosity of the air-water mixture in the droplet layer. According to the two boundaries of the droplet layer at $y_{90}$ and $y_{99}, \mu_{\mathrm{m}}$ can be estimated as $\mu_{m} \approx 0.9 \mu_{w}+0.1 \mu_{a}$, where $\mu_{w}$ and $\mu_{a}$ are the dynamic viscosities of water and air, respectively. Then, the $\tau_{\mathrm{m}}$ of the entire droplet layer dragging the air flow is $\tau_{\mathrm{m}} \approx \mu_{\mathrm{m}}+\left(0.98 u_{\mathrm{fs}} / \delta_{\mathrm{w}}\right)$. For an engineering view, standard computations of free surface velocity $u_{\mathrm{fs}}$ are widely used $[13,34]$. Based on the complete average velocity profile of a 


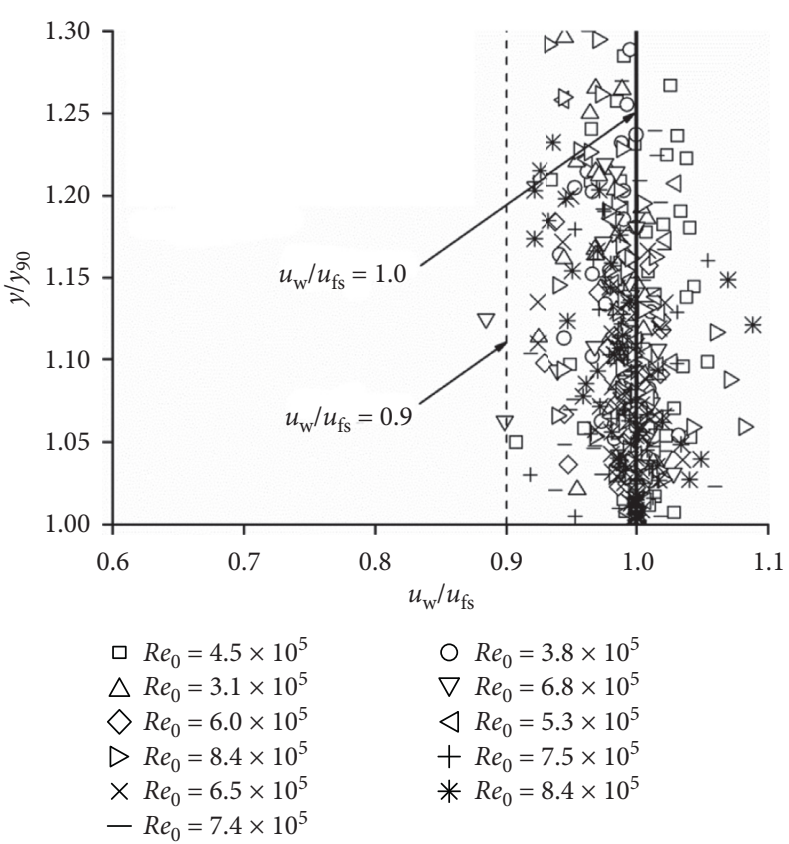

(a)

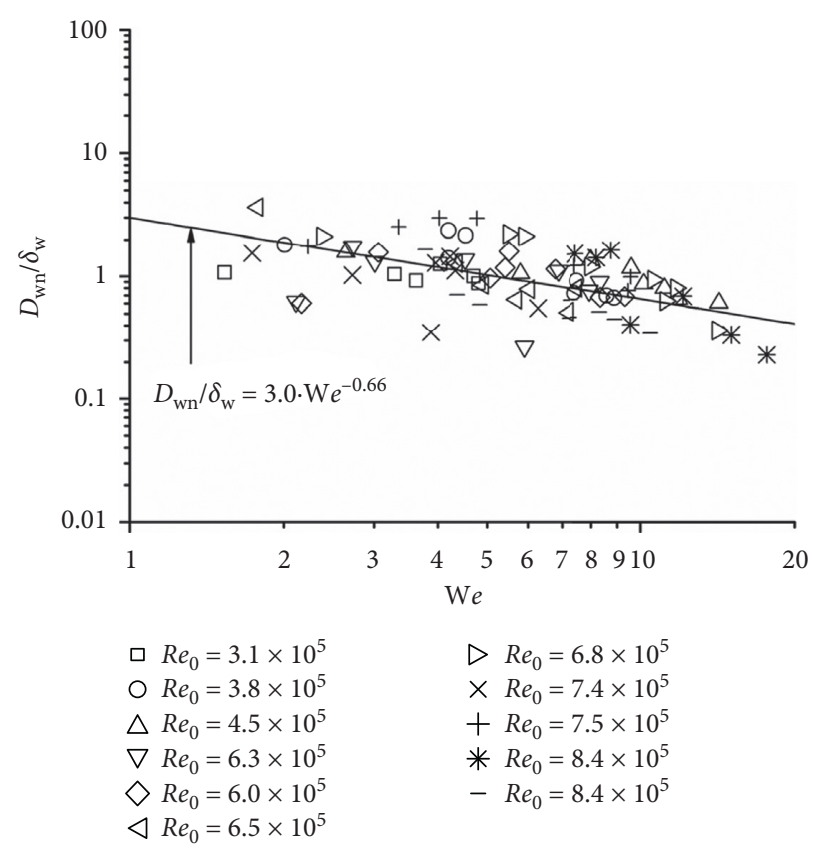

(b)

Figure 9: (a) Droplet velocity distributions in droplet layers and (b) a function of Weber number.

logarithmic wall-dominated term [35], in which the entire droplet layer is considered to be in the "wall-dominated condition," the air velocity distribution is

$$
\frac{u_{a}}{u_{*}}=\frac{1}{k} \ln \left(\frac{y_{a}}{k_{w}}\right)+\frac{1}{k}(1+6 \Pi)\left(\frac{y_{a}}{\delta_{a}}\right)^{2}-\frac{1}{k}(1+4 \Pi)\left(\frac{y_{a}}{\delta_{a}}\right)^{3}+B,
$$

where $k$ is the von Karman constant (0.41); the constant $B$ is $8.5 ; \Pi \approx 0.2$ is the wake parameter [36]; $k_{\mathrm{w}}$ is the equivalent roughness height of the droplet layer inducing the air layer generation, and can be represented by the droplet size characteristics, ranging from 1 to $10 \mathrm{~mm}$ as per the experimental results; and the shear velocity $u_{*}=u^{*}=\left(\tau_{\mathrm{m}} / \rho_{\mathrm{m}}\right)^{0.5}$, in which the air-water mixture density $\rho_{\mathrm{m}}$ is estimated by $\rho_{\mathrm{m}}=0.9 \rho_{\mathrm{w}}+0.1 \rho_{\mathrm{a}}$. Using the boundary condition that $u_{\mathrm{a}} \approx u_{\mathrm{fs}}$ at $y_{\mathrm{a}}=0$, equation (7) becomes

$$
\frac{u_{f s}}{u_{*}}=\frac{1}{k} \ln \left(\frac{\delta_{a}}{k_{w}}\right)+\frac{2 \Pi}{k}+B \equiv\left(\frac{2}{C_{f}}\right)^{0.5} .
$$

Thus, an analytic solution for the air boundary layer thickness $\delta_{\mathrm{a}}$ is obtained. By fitting the air velocity to a powerlaw $[14,37]$,

$$
\frac{u_{f s}-u_{a}}{u_{f s}}=\left(\frac{y_{a}}{\delta_{a}}\right)^{1 / n}
$$

where the exponent factor $n$ of the power profile can be determined from the wake-wall model.

In hydraulic engineering applications, the air layer induced by high-speed self-aerated flows can be assessed using equations (8) and (9). Valero and Bung [14] pioneered the theoretical and experimental study of the interfacial air layer on spillways. Given a certain droplet layer thickness, the thickness of the air layer increases with the generalized droplet roughness $k_{\mathrm{w}}$. Compared to the measured $\delta_{\mathrm{a}}$ in Figure 10(a), the boundary layer growth is well satisfied using $\delta_{\mathrm{w}}=0.005-0.010 \mathrm{~m}$ with a $k_{\mathrm{w}}$ ranging from 1 to $2 \mathrm{~mm}$. For an average flow depth of $0.026-0.034 \mathrm{~m}$, the predicted droplet layer is about $15-30 \%$ of the flow depth, fitting the measured droplet layer growth (Figure 5). Due to the low instability fluctuations and weak entrapped air around the self-aeration onset region, it can be deduced that the droplet size in the thin droplet layer is small. Using $\delta_{\mathrm{w}}=0.005 \mathrm{~m}$ and $\delta_{\mathrm{w}}=0.010 \mathrm{~m}$, the exponent value corresponds to $n=4.27$ and 6.46 , respectively, which are in agreement with $n=5.15$ from the air velocity measurements, as shown in Figure 10(b). Furthermore, reasonable air velocity profiles determined using $n=4.27-6.46$ approximately fit the experimental data. Consequently, in terms of the air layer prediction based on the air-water mixture in the free surface region, the improved model permits the determination of $\delta_{\mathrm{a}}$ and $n$ values that are in agreement with observations for a given air-water flow condition.

For self-aerated open channel flows, the effects of water droplet layer thickness on air layer growth and the shape of the air velocity profile are summarized in Figure 11 . As the droplet layer develops, $\delta_{\mathrm{a}}$ increases considerably with $\delta_{\mathrm{w}}$. A larger droplet layer roughness $k_{\mathrm{w}}$, characterized by the size of the air-water structures, strengthens the air layer development resulting in a larger value of $\delta_{\mathrm{a}}$. This agrees with the findings of previous investigations indicating that the height of the air layer can reach over four times the bulk flow depth, mainly because the boundary layer shape is related to the fluid pressure gradient, as noted by White [35], and droplet layer growth results in a high $\delta_{\mathrm{a}}$ development rate. Additionally, the 


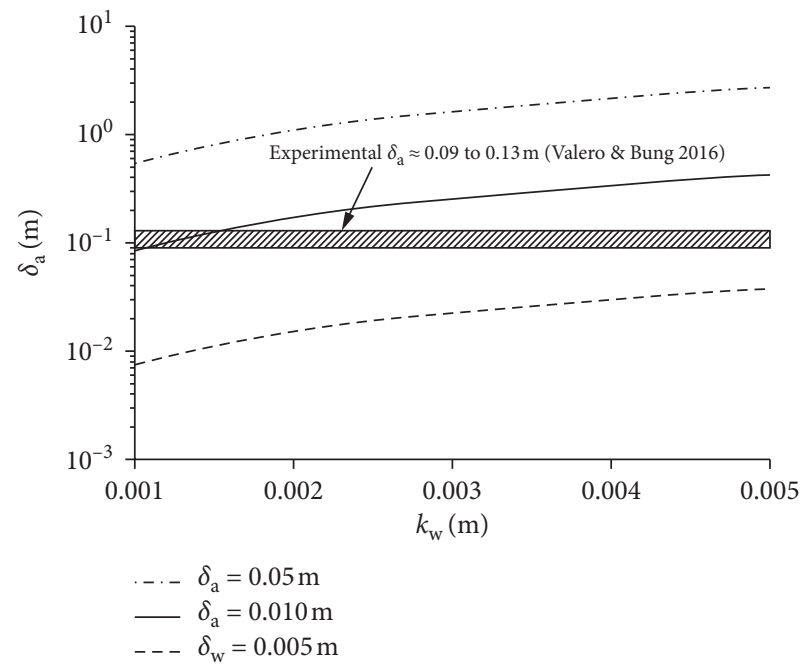

(a)

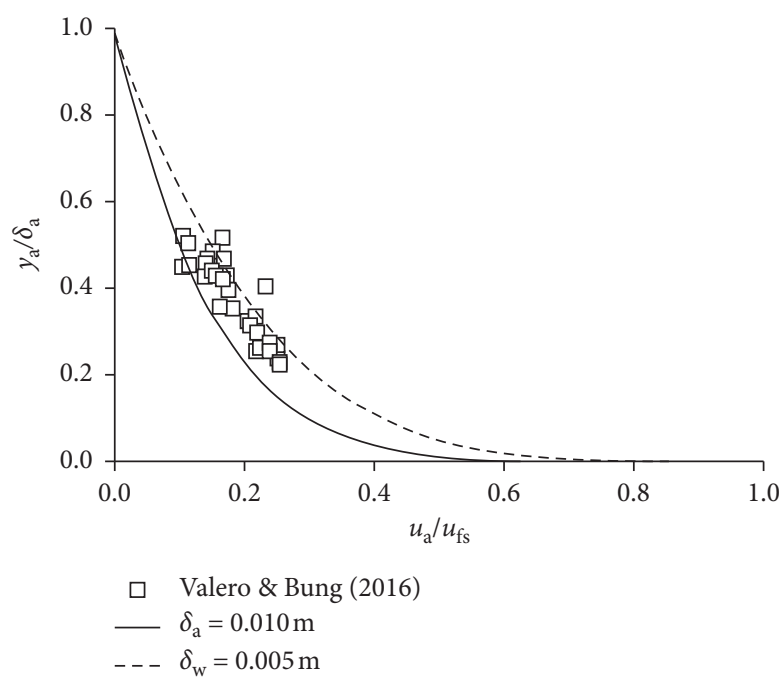

(b)

Figure 10: Predictions and comparisons of air layer (a) thickness and (b) velocity distribution.

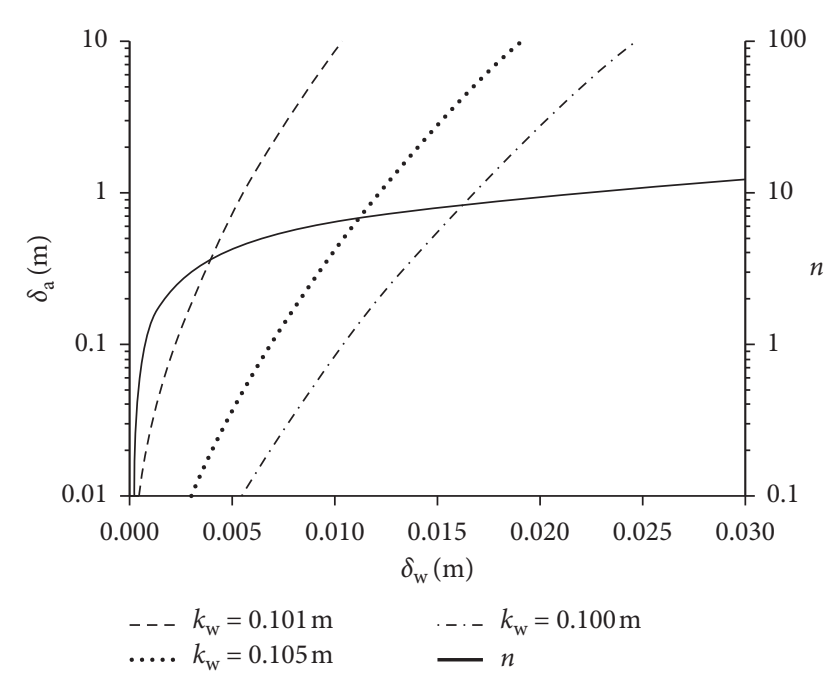

FIGURE 11: Effects of layer thickness and roughness on air layer factors.

exponent of the air velocity profile $n$ is related to the $\delta_{\mathrm{w}}$ of the developing droplet layer thickness and is independent of the air-water structures in the droplet boundary layer. An increase in either the droplet layer thickness or exponent of the air velocity profile leads to an increase in the air movement induced by the free surface of the air-water layer along the direction of flow development downstream. Finally, these findings indicate that not only is the air layer growth rate high over the initial distance of droplet layer generation, which in agreement with the general development of chute flow, but also that the general expression for the exponent $n$ tends to level off for fully developed self-aerated flows.

For the self-aeration development in high-speed open channel flows, the entire mixture process of air-water particles contains two parts, that is, air bubble diffusion in the main bulk flow and water droplet above the free surface $\left(y_{90}\right)$. Using the free surface boundary $\left(y_{90}\right)$ at which the air concentration is 0.9 , the previous studies $[19,24]$ indicate that as the channel slope increase, the process for bottom self-aeration develops more pronounced, while the mean cross-sectional air concentration is only a function of the channel slope for uniform region of self-aerated flows. The present study is to investigate the detailed features of droplet layer development above the free surface. The objective is to provide an improved approach for the air velocity distribution affected by the droplet layer above the highly aerated area where $C>0.90$. Thus, the effect of channel slope should be considered differently. Firstly, the uniform trend of droplet layer growth is independent of channel slope (Figure 4). The agreement with a stepped spillway experimental test with $\alpha=26.6^{\circ}$ for air velocity profile prediction indicates that a universal model can be constructed on the basis of air boundary layer thickness.

The present study also show the effect of aeration development distance on the microscopic droplet size distribution above the free surface, and no clear trend is observed for different flow Reynolds number $\operatorname{Re}_{0}$. The quick variation of droplet frequency distribution in $0 \leq\left(x / h_{0}\right) \leq 100$ indicates that the effect of aeration process on the detailed air boundary layer is pronounced in the initial aeration distance (Figures 7 and 8), and the air-water mixture gets uniform as the aeration develops downstream. Besides, the water droplet size in the upper region of droplet layer closed to air flow layer is stable and independent of $\mathrm{Re}_{0}$, approximately $d_{\mathrm{wm}} / \delta_{\mathrm{w}}=0.5$. It can be deduced that the effect of channel slope on equivalent roughness height of the droplet layer for air velocity distribution prediction is weak.

As a next step, the effect of the channel slope on the detailed air-water mixture and air boundary layer should be investigated. Based on the data collection, including the airwater velocity, droplet quantity, and size, an updated theoretical model describing the air boundary layer will be prepared for further analysis. 


\section{Conclusions}

In this study, air-water flow measurements were used to quantify the relative contributions of the drag effect on the air layer induced above the highly aerated free surface of open channel flows. In the droplet mixture region above the bulk flow, the air-water mixture was characterized by different quantities and sizes of water-dominated flow structures. With the development of self-aeration downstream, the droplet layer thickness was observed to increase independently of other flow conditions. The power profile of the droplet frequency became smooth in the upper region of the droplet layer, in which the droplet size remains approximately a half of droplet layer thickness. This is consistent with the classical model for droplet size moving above the boundary-free surface of wall jets. Based on the classical boundary layer theory, the effects of droplet layer thickness and droplet roughness was introduced to propose an improved approach for determining the parameters of the power-law for air velocity profiles. The analytical solution of the boundary layer equations yielded an explicit expression for the air velocity profile induced by the droplet layer. Finally, the predicted results were shown to be in reasonable agreement with previously obtained experimental data in the literature. The findings of the study are expected to be useful in modeling the free surface region of open channel flows.

\section{Data Availability}

The data used to support the findings of this study are included within the article.

\section{Conflicts of Interest}

The authors declare that they have no conflicts of interest.

\section{Acknowledgments}

The work was completed within the research projects funded by the National Natural Science Foundation of China (grant nos. 51979183 and 51939007) and Sichuan Science and Technology Program (grant nos. 2019JDTD0007 and 2020YJ0320).

\section{References}

[1] S. Smolentsev and R. Miraghaie, "Study of a free surface in open-channel water flows in the regime from "weak" to "strong" turbulence," International Journal of Multiphase Flow, vol. 31, no. 8, pp. 921-939, 2005.

[2] D. Garg, A. Longo, and P. Papale, "Modeling free surface flows using stabilized finite element method," Mathematical Problems in Engineering, vol. 2018, Article ID 6154251, 2018.

[3] M. A. André and P. M. Bardet, "Velocity field, surface profile and curvature resolution of steep and short free surface waves," Experiment in Fluids, vol. 55, no. 4, 2014.

[4] I. Calmet and J. Magnaudet, "Statistical structure of highReynolds-number turbulence close to the free surface of an open-channel flow," Journal of Fluid Mechanics, vol. 474, pp. 355-378, 2003.
[5] M. Brocchini and D. H. Peregrine, "The dynamics of strong turbulence at free surfaces. Part 1. Description," Journal of Fluid Mechanics, vol. 449, pp. 225-254, 2001.

[6] L. G. Straub and A. G. Anderson, "Experiments on selfaerated flow in open channels," Journal of the Hydraulics Division, ASCE, vol. 84, no. 7, pp. 1-35, 1958.

[7] S. C. Wilhelms and J. S. Gulliver, "Bubbles and waves description of self-aerated spillway flow," Journal of Hydraulic Research, vol. 43, no. 5, pp. 522-531, 2005.

[8] J. George, F. Minel, and M. Grisenti, "Physical and hydrodynamical parameters controlling gas-liquid mass transfer," International Journal of Heat and Mass Transfer, vol. 37, no. 11, pp. 1569-1578, 1994.

[9] D. Dabiri and M. Gharib, "Experimental investigation of the vorticity generation within a spilling water wave," Journal of Fluid Mechanics, vol. 330, pp. 113-139, 1997.

[10] M. Brocchini and D. H. Peregrine, "The dynamics of strong turbulence at free surfaces. Part 2. Free-surface boundary conditions," Journal of Fluid Mechanics, vol. 449, pp. 255-290, 2001.

[11] O. Castro-Orgaz, "Hydraulics of developing chute flow," Journal of Hydraulic Research, vol. 47, no. 2, pp. 185-194, 2009.

[12] S. B. Pope, Turbulent Flows, Cambridge University Press, Cambridge, UK, 2000.

[13] O. Castro-Orgaz, "Velocity profile and flow resistance models for developing chute flow," Journal of Hydraulic Engineering, vol. 136, no. 7, pp. 447-452, 2010.

[14] D. Valero and D. B. Bung, "Development of the interfacial air layer in the non-aerated region of high-velocity spillway flows. Instabilities growth, entrapped air and influence on the selfaeration onset," International Journal of Multiphase Flow, vol. 84, pp. 99-107, 2016.

[15] W. H. Hager, "Uniform aerated chute flow," Journal of Hydraulic Engineering, vol. 117, no. 4, pp. 528-533, 1991.

[16] D. B. Bung, "Non-intrusive detection of air-water surface roughness in self-aerated chute flows," Journal of Hydraulic Research, vol. 51, no. 3, pp. 322-329, 2013.

[17] D. B. Bung and D. Valero, "Optical flow estimation in aerated flows," Journal of Hydraulic Research, vol. 54, no. 5, pp. 575-580, 2016.

[18] W. Wei, W. Xu, J. Deng, Z. Tian, and F. Zhang, "Free-surface air entrainment in open-channel flows," Science China Technological Sciences, vol. 60, no. 6, pp. 893-901, 2017.

[19] W. Wei, J. Deng, and F. Zhang, "Development of self-aeration process for supercritical chute flows," International Journal of Multiphase Flow, vol. 79, pp. 172-180, 2016.

[20] W.-r. Wei, W.-l. Xu, J. Deng, Z. Tian, and F.-x. Zhang, "Experimental study of air-water interface properties in selfaerated flows," Journal of Hydrodynamics, vol. 31, no. 5, pp. 940-948, 2019.

[21] J. M. Killen, The surface characteristics of self-aerated flow in steep channels, Ph.D. Thesis, University of Minnesota, Minneapolis, MN USA, 1968.

[22] P. Cain and I. R. Wood, "Measurements of self-aerated flow on a spillway," Journal of the Hydraulics Division, ASCE, vol. 107, no. 11, pp. 1425-1444, 1981.

[23] F. F. Campbell, R. G. Cox, and M. B. Boyd, "Boundary layer development and spillway energy loss," Journal of the Hydraulics Division, ASCE, vol. 91, no. 3, pp. 149-163, 1965.

[24] I. R. Wood, "Uniform region of self-aerated flow," Journal of Hydraulic Engineering, vol. 109, no. 3, pp. 447-461, 1983.

[25] Z. Dai, L. P. Hsiang, and G. Faeth, "Spray formation at the free surface of turbulent bow sheets," in Proceedings of the 21th 
Symposium on Naval Hydrodynamics, vol. 1, pp. 197-211, Washington DC, USA, 1996.

[26] T. Sarpkaya and C. F. Merrill, "Spray generation from turbulent plane water wall jets discharging into quiescent air," AIAA Journal, vol. 39, no. 7, pp. 1217-1229, 2001.

[27] M. Popescu and S. Johansen, "The study of the sea freezing spray droplet and their contribution to ice accretion," in Proceedings of 4th AIAA Atmospheric and Space Environments Conference, New Orleans, LA, USA, 2012.

[28] C. Merrill and T. Sarpkaya, "Spray formation at the free surface of a liquid wall jet," in Proceedings of 36th AIAA Aerospace Sciences Meeting and Exhibit, Reston, NV, USA, 1998.

[29] P.-K. Wu and G. M. Faeth, "Onset and end of drop formation along the surface of turbulent liquid jets in still gases," Physics of Fluids, vol. 7, no. 11, pp. 2915-2917, 1995.

[30] Z. Dai, W.-H. Chou, and G. M. Faeth, "Drop formation due to turbulent primary breakup at the free surface of plane liquid wall jets," Physics of Fluids, vol. 10, no. 5, pp. 1147-1157, 1998.

[31] S. Komori, H. Ueda, F. Ogino, and T. Mizushina, "Turbulence structure and transport mechanism at the free surface in an open channel flow," International Journal of Heat and Mass Transfer, vol. 25, no. 4, pp. 513-521, 1982.

[32] M. Ishii and T. Hibiki, Thermo-fluid Dynamics of Two-phase Flow, Springer, Berlin, Germany, 2010.

[33] D. B. DeGraaf and J. K. Eaton, "Reynolds number scaling of the flat-plate turbulent boundary layer," Journal of Fluid Mechanics, vol. 422, pp. 319-346, 2000.

[34] H. T. Falvey, "Air-water flow in hydraulic structures, Water and Power Resources Service," Engineering Monograph, vol. 41, 1980.

[35] F. M. White, Viscous Fluid Flow, McGraw-Hill, New York, NY, USA, 1991.

[36] B. A. Kironoto and W. H. Graf, "Turbulence characteristics in rough uniform open-channel flow," Proceedings of the Institution of Civil Engineers - Water Maritime and Energy, vol. 106, no. 4, pp. 333-344, 1994.

[37] J. Yazdi and A. R. Zarrati, "An algorithm for calculating air demand in gated tunnels using a 3D numerical model," Journal of Hydro-Environment Research, vol. 5, no. 1, pp. 3-13, 2001. 